\title{
Planung und Vorbereitung von Eingriffen im Bereich des Ellenbogengelenks
}

\author{
Tobias Nowak, Sven-Oliver Dietz, Pol M. Rommens
}

\section{Zusammenfassung}

Die Planung und Vorbereitung operativer Eingriffe am Ellenbogengelenk umfasst die Anamneseerhebung, die körperliche Untersuchung, die Durchführung der notwendigen Bildgebung und deren korrekte Interpretation, die Auswahl des geeigneten Operationsverfahrens inkl. geeigneter Implantate und schließlich die korrekte Lagerung des Patienten im Operationssaal.

\section{Planning and Preparation of Operations in the Elbow Region}

The planning and preparation of surgical interventions on the elbow joint encompass the case history, physical examination, performance of the necessary imaging studies and their correct interpretation, the choice of a suitable operative procedure and appropriate implants as well as, last but not least, the correct positioning of the patient in the operating room.

\section{Einleitung}

Der Satz: „Failing to plan is planning to fail“ [1] hat am Ellenbogengelenk besondere Bedeutung. Verschiedene Verletzungen und Erkrankungen des Ellenbogengelenks erfordern spezifische präoperative diagnostische Maßnahmen. Deren Kenntnis und korrekte Interpretation führt zur richtigen Diagnose. Die Diagnose führt zur Entscheidung für einen operativen Eingriff. Für diesen Eingriff muss sich der Operateur eine Strategie zurechtlegen.

Diese beinhaltet die korrekte Lagerung, die Wahl des geeigneten Verfahrens und Zugangs sowie die richtigen Implantate und bereits präoperativ die Planung der postoperativen Weiterbehandlung des Patienten.

Die korrekte Planung ist auch für die Kommunikation mit den beteiligten Fachdisziplinen zwingend erforderlich. Gemeinsam mit dem Anästhesisten können die Narkoseform und die postoperative Schmerztherapie optimiert werden. Das frühzeitige Festlegen auf ein bestimmtes operatives Verfahren erleich-

OP-JOURNAL 2011; 27: 26-33

(c) Georg Thieme Verlag KG Stuttgart · New York DOI http://dx.doi.org/10.1055/s-0030-1271079 tert es dem OP-Pflegepersonal, die Operation perfekt vorzubereiten und das benötigte Material vorzuhalten.

Die Planung und Vorbereitung operativer Eingriffe am Ellenbogengelenk umfasst die Anamneseerhebung, die körperliche Untersuchung, die Durchführung der notwendigen Bildgebung und deren korrekte Interpretation, die Auswahl des geeigneten Operationsverfahrens inklusive geeigneter Implantate und schließlich die korrekte Lagerung des Patienten im Operationssaal.

In der folgenden Arbeit soll auf diese Punkte eingegangen werden:

1. Anatomie des Ellenbogengelenks

2. Untersuchung des Patienten

3. Bildgebung am Ellenbogen

4. Lagerung des Patienten

5. Planung und Vorbereitung einer Ellenbogenarthroskopie

6. Planung einer Osteosynthese am Ellenbogen

\section{Anatomie des Ellenbogengelenks}

Das Ellenbogengelenk besteht aus $3 \mathrm{Ge}-$ lenkbereichen, die in einer gemeinsamen Gelenkkapsel liegen: das Humeroulnargelenk, das Humeroradialgelenk und das proximale Radioulnargelenk (Abb. 1). Da die Gelenkkapsel dünn und schlaff ist, strahlen von ventral der M. brachialis und von dorsal die Mm. triceps brachii und anconeus in die Kapsel ein, um ein Einklemmen zwischen den Gelenkkörpern zu verhindern (Abb.2). Die beiden Kollateralbänder sind Verdickungen der medialen und lateralen Kapselanteile. Beide Bänder haben proximal einen schmalen Ursprung am Humerus und setzen distal verbreitert fächerförmig an (Abb. 3). Dadurch ist in jeder Gelenkposition immer ein Teil der Kollateralbänder zur Führung und Sicherung des Gelenks angespannt. Das mediale Kollateralband entspringt am Epicondylus ulnaris humeri und zieht mit 2 starken Faserportionen zur Basis des Processus coronoideus und zum Olekranon. Vom Epicondylus radialis humeri entspringt das Lig. collaterale radiale und zieht zum Radiuskopf, wo es in das Lig. anulare übergeht. Das Lig. anulare und der Lig. collaterale radiale dienen zusammen als Ursprungsfläche für die oberflächlichen Handgelenksextensoren und auch für den M. supinator. Außerdem entspringt das laterale ulnare Kollateralband (LUCL) vom radialen Epikondylus und zieht zur lateralen Ulna. Dieses Band sichert das Humeroulnargelenk und gilt als der hauptsächliche laterale Stabilisator. Es ist sowohl in Flexion als auch in Extension gespannt. In der Tiefe zwischen M. brachialis und M. brachioradialis gelangen der $\mathrm{N}$. radialis und die begleitende Arterie in die Ellenbeuge. Der N. radialis teilt sich hier in einen oberflächlichen und einen tiefen Ast. Der oberflächliche Ast folgt nach distal dem M. brachioradialis und schließt sich der A. radialis an. Der tiefe Ast durchbohrt den M. supinator und schlingt sich um den Radiuskopf und gelangt im weiteren Verlauf in die Extensorenloge.

Im Humeroulnargelenk artikuliert die Trochlea humeri mit der Incisura trochlearis ulnae. Beide Gelenkkörper sind schlüssig ineinander gefügt und bilden so ein knochengeführtes Gelenk. Das Humeroradialgelenk ist ein Kugelgelenk, 
das aufgrund des straffen Kollateralbandapparats, welcher nach distal in das Lig. anulare radii übergeht und den Radiuskopf an der Ulna fixiert, auf 2 Freiheitsgrade beschränkt bleibt. Übrig bleiben die Scharnierbewegung, bei der der Radiuskopf auf dem Capitulum humeri gleitet und die Drehbewegung, bei der die Speiche innerhalb des Lig. anulare auf dem Capitulum dreht. Im proximalen Radioulnargelenk dreht der Radiuskopf geführt durch das Lig. anulare in der Incisura radialis ulnae. Durch das Zusammenwirken der 3 Gelenke sind in jeder Beuge- und Streckstellung gleichzeitig Drehbewegungen des Radius um die Ulna möglich.

\section{Klinische Untersuchung des Ellenbogengelenks}

\section{Anamnese}

Zu Beginn der Untersuchung sollte immer eine ausführliche Anamnese durchgeführt werden. Hierbei werden Fragen nach vorausgegangenen Verletzungen, Systemerkrankungen, beruflicher und sportlicher Betätigung und Unfallhergang gestellt. Leitsymptom bei Unfallfolgen und degenerativen Erkrankungen ist der Schmerz. Insbesondere bei chronischen Beschwerden ist ein genaues Befragen über die Lokalisation, den bevorzugten Zeitpunkt und auch über die Schmerzqualität von großer Wichtigkeit. Bei einem akuten Trauma bringt die Beschreibung des Unfallmechanismus Informationen, ob eine direkte oder indirekte Verletzung vorliegt. Ein Ziel der Traumaanamnese sollte auch sein, herauszufinden, ob ein adäquates Trauma vorgelegen hat.

\section{Inspektion}

Die Untersuchung des Ellenbogengelenks erfolgt am sitzenden oder stehenden Patienten. Zunächst wird der Ellenbogen bei vollständig entkleidetem Oberkörper inspiziert. Hierbei sollte auf Stellungsvarianten und Konturveränderungen geachtet werden.

In gestreckter und supinierter Stellung bilden die Längsachsen von Ober- und Unterarm einen nach lateral offenen Winkel im Sinne eines Valgus. Bei Männern beträgt dieser Winkel ca. $5^{\circ}$, bei Frauen können als Normvarianten Winkel zwischen $5^{\circ}$ und $15^{\circ}$ vorkommen. Fehlstellungen im Sinne eines Cubitus valgus können durch vorangegangene Frakturen des Epicondylus radialis be-

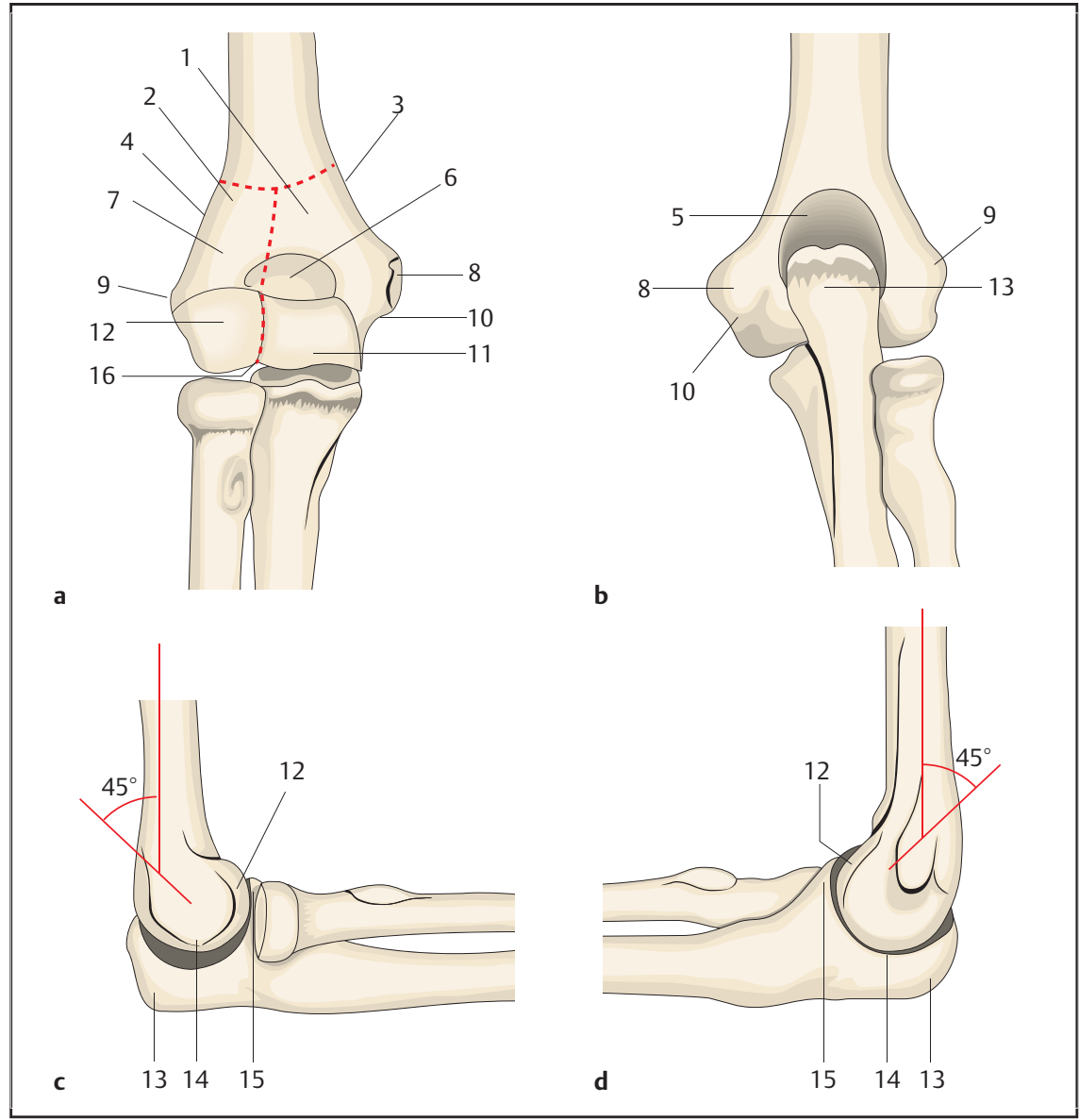

Abb. 1 a bis d Knöcherne Anteile des Ellenbogengelenks: Rechter Ellenbogen: Ansicht von ventral (a) und von dorsal (b) bei ausgestrecktem Ellenbogen; Ansicht von seitlich (c) und von medial (d) bei $90^{\circ}$ gebeugtem Ellenbogen. 1: mediale Kondyle; 2: laterale Kondyle; 3: Crista supacondylaris medialis; 4: Crista supracondylaris lateralis; 5: Fossa olecrani; 6: Fossa coronoidea; 7: Fossa radialis; 8: Epicondylus medialis; 9: Epicondylus lateralis; 10: Sulcus nervi ulnaris; 11: Trochlea; 12: Capitulum humeri: 13: Olecranon; 14: Incisura trochlearis (trochlear notch); 15: Processus coronoideus; 16: Sulcus capitullotrochlearis.

dingt sein. Frakturen im Kindesalter können varische Fehlstellungen verursachen.

Bei ausgedehnten Gelenkergüssen wird der Ellenbogen in ca. $45^{\circ}$ Flexion gehalten, da in dieser Stellung das Fassungsvermögen der Gelenkkapsel am größten ist. Ursächlich sind hierfür meist Frakturen oder Quetschverletzungen. Wenn zusätzlich Rötung oder Überwärmung vorliegen, sollte an eine Gelenkentzündung oder auch an eine Bursitis gedacht werden.

Krepitationen bei der Flexion können durch Frakturen, Arthrose, freie Gelenkkörper, eine verdickte Synovia oder eine Bursa hervorgerufen werden.

Die knöchernen Landmarks sind die Spitze des Olekranon, der mediale und der laterale Epikondylus. Der N. ulnaris ist medial im Sulcus ulnaris zwischen
Epicondylus ulnaris und dem Olekranon zu tasten. Des Weiteren entspringen an der Innenseite des Ellenbogens die Handgelenksflexoren und -pronatoren. Hier lässt sich oftmals eine Druckschmerzhaftigkeit provozieren. Dorsal über dem Ellenbogen lassen sich das Olekranon und der M. triceps brachii tasten. Lateral tastet man in Verlängerung des Epicondylus radialis humeri den Radiuskopf [2]. Die Durchblutung sollte zum Ausschluss von Gefäßverletzungen (z.B. A.-radialisLäsion) überprüft werden.

\section{Bewegungsumfänge}

Das Bewegungsausmaß des Ellenbogens setzt sich aus 4 Bewegungsrichtungen (Extension/Flexion und Pronation/Supination) zusammen.

Die Beweglichkeitsprüfung wird nach der Neutral-Null-Methode durchgeführt. 
Ein normales Bewegungsausmaß beträgt in Extension und Flexion einen Bereich von 0 bis $150^{\circ}$. Bei Frauen kann eine physiologische Überstreckbarkeit bis $\mathrm{zu} 10^{\circ}$ vorliegen. Eine darüber hinausreichende Überstreckung spricht für eine Gelenkinstabilität oder Hyperlaxität. Ein Streckdefizit besteht häufig bei Arthrose, oft auch in Verbindung mit freien Gelenkkörpern. Weitere Ursachen hierfür können aseptische Knochennekrosen, eine Verklebung der Recessus nach Unfällen, vorangegangenen Operationen und letztlich auch eine rheumatoide Arthritis darstellen.

Die Prüfung der Umwendbewegung (Pronation/Supination) erfolgt in Adduktion des Schultergelenks und $90^{\circ}$ flektiertem Ellenbogen. Hierbei zeigt sich physiologischerweise ein Bewegungsausmaß von etwa jeweils $80-90^{\circ}$. Die Rotation der Speiche um die Elle sollte insgesamt etwa $160^{\circ}$ betragen.

\section{Funktionelle Tests}

\section{Stabilität}

Zur Überprüfung der Bandstrukturen übt der Untersucher einen Varus- und Valgusstress auf den Unterarm bei dorsal fixiertem Ellenbogen aus. In Extension sind die vorderen Anteile des ulnaren Kollateralbands angespannt und in Flexion die dorsalen. Der mediale Bandapparat wird in leichter Flexion und Supination überprüft. Eine posterolaterale Instabilität wird bei etwa $30^{\circ}$ Flexion, Supination und durch Ausübung von axialer Kompression geprüft. Liegt eine posterolaterale Instabilität vor, kommt es zu einer sichtbaren und tastbaren Subluxation im Humeroradialgelenk. Dasselbe Phänomen kann auch beim liegenden Patienten in Schulterextension getestet werden (sog. Pivot-Shift-Test).

\section{Epikondylopathien}

Bei einer radialen Epikondylopathie besteht eine lokale Druckschmerzhaftigkeit über dem Usrprungsbereich der Handextensoren am radialen Epikondylus. Schmerzen bestehen auch bei kräftigem Händedruck, bei forcierter Dorsalextension der geballten Faust und beim Halten oder Heben schwerer Gegenstände mit gestrecktem Arm. Bei der ulnaren Epikondylopathie lässt sich eine Druckdolenz medial im Ursprungsbereich der Handbeuger finden. Außerdem äußert der Patient Schmerzen bei forcierter Palmarflexion der geballten Faust [3].

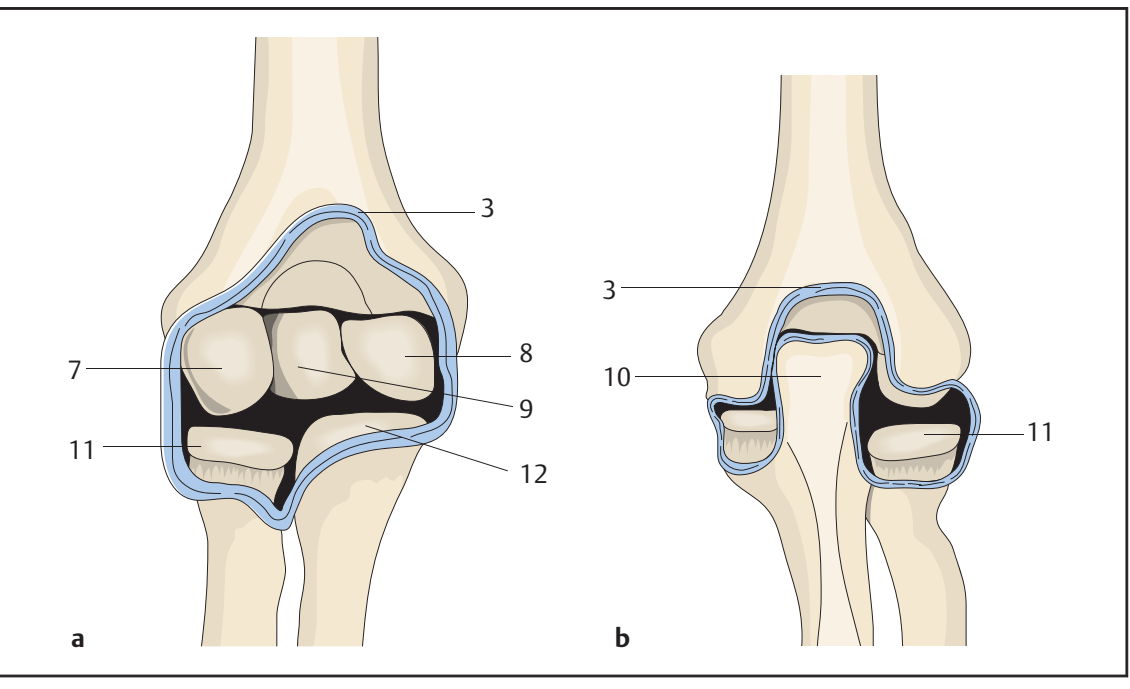

Abb. 2 a und $\mathbf{b}$ Die Gelenkkapsel des Ellenbogengelenks: Rechter Ellenbogen in Extension von ventral (a) und von dorsal (b). 3: Gelenkkapsel; 7: Capitulum humeri; 8: Trochlea humeri; 9: Sulcus capitullotrochlearis; 10: Olecranon; 11: Caput radii; Processus coronoideus.

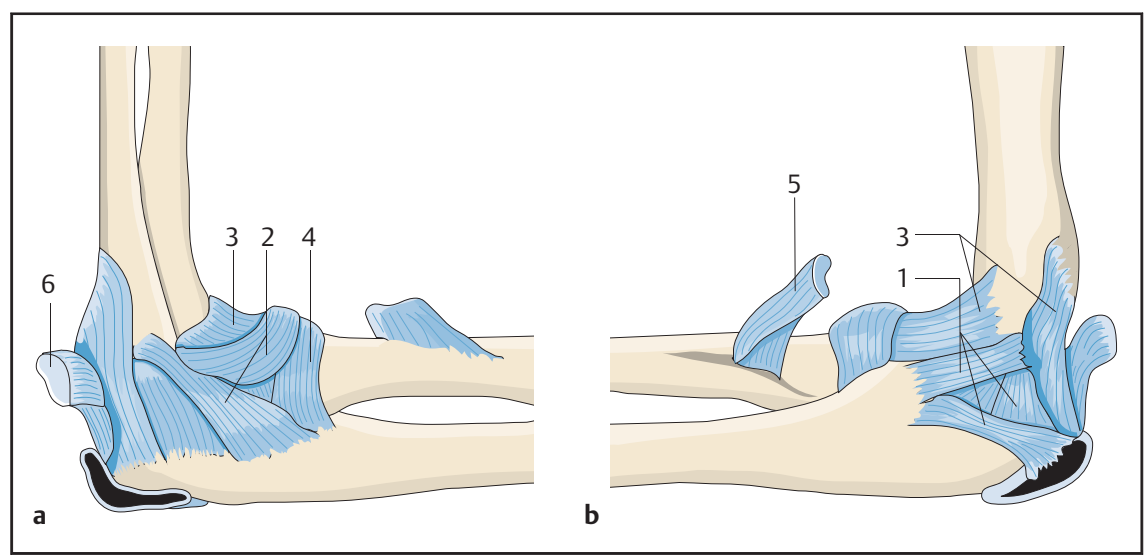

Abb. 3 a und $\mathbf{b}$ Bandstrukturen des Ellenbogengelenks: Rechter Ellenbogen in $90^{\circ}$ Beugung von lateral (a) und medial (b). 1: Lig. collaterale ulnare (a: vorderer Anteil, b: hinterer Anteil); 2: Lig. collaterale radiale (a: vorderer Anteil, b: hinterer Anteil, c: Lig. obliquum); 3: Gelenkkapsel; 4: Lig. anulare radii; 5: Tendo $\mathrm{m}$. bicipis brachii; 6: Tendo $\mathrm{m}$. tricipitis brachii.

\section{Neurologische Untersuchung}

Bei der neurologischen Untersuchung werden Motorik, Reflexe und Sensibilität untersucht.

\section{Motorik}

Zur Untersuchung der Motorik wird die Muskelfunktion in den Hauptbewegungsrichtungen Flexion, Extension, Pro- und Supination geprüft.

Die Flexion wird im Wesentlichen durch den $\mathrm{M}$. brachialis und den M. biceps brachii durchgeführt. Beide Muskeln werden vom N. musculocutaneus $(C 5, C 6)$ innerviert.
Zur Prüfung der Muskelfunktion flektiert der Patient den Unterarm gegen den Widerstand des Untersuchers.

Die Extension des Ellenbogengelenks erfolgt durch den M. triceps brachii. Dieser wird durch den $\mathrm{N}$. radialis (C7) innerviert. Die Testung erfolgt, indem der Patient gegen den Widerstand des Untersuchers das Ellenbogengelenk streckt.

Die Umwendbewegungen werden bei gebeugtem Ellenbogen mit der Hand in Neutralstellung untersucht. Die Pronation wird durch die Mm. pronator teres und pronator quadratus initiiert. Ersterer wird vom N. medianus (C6), Letzterer vom $\mathrm{N}$. interosseus antebrachii anterior (C8, Th1) innerviert. Die Supination er- 


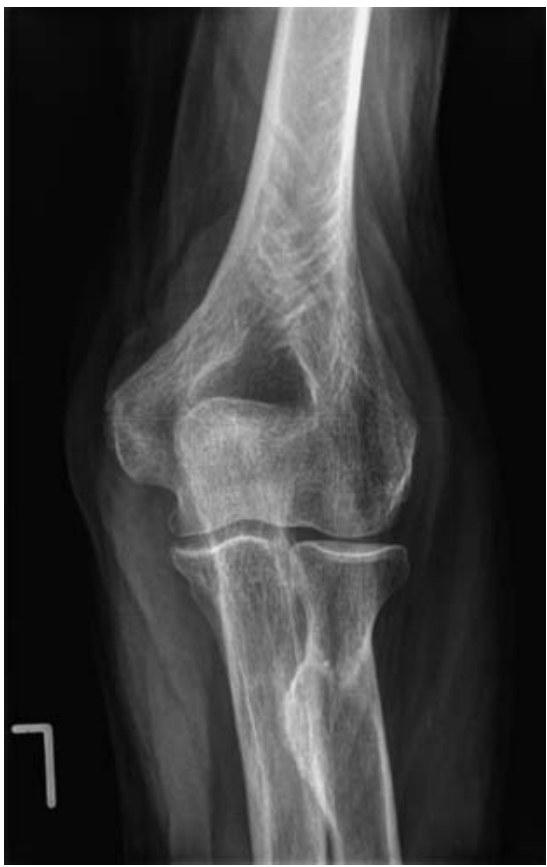

Abb. 4 A.-p. Aufnahme des Ellenbogens (am Beispiel eines linken Ellenbogens).

folgt durch die Mm. biceps brachii und M. supinator (N. musculocutaneus $\mathrm{C} 5$, C6; N. radialis C6) [4].

\section{Reflexe}

Am Ellenbogengelenk werden die Reflexe von 3 Muskeln untersucht.

Der Bizepssehnenreflex (C5) erfolgt am leicht gebeugten und leicht pronierten Ellenbogen. Der Untersucher klopft mit dem Reflexhammer auf seinen Daumen, der auf dem distalen Anteil der Bizepssehne liegt. Als Reflexantwort kommt es zu einer Beugung und Supination im Ellenbogengelenk.

Der Brachioradialisreflex (C6) wird in gleicher Position wie der Bizepssehnenreflex geprüft. Der Untersucher klopft mit dem Reflexhammer auf die Brachioradialissehne am distalen Radius.

Beim Trizepssehnenreflex (C7) erfolgt als Reflexantwort eine Streckung im Ellenbogengelenk. Hierfür wird bei gebeugtem Ellenbogen mit dem Reflexhammer auf die olekranonnahe Trizepssehne geklopft.

\section{Sensibilität}

Die Untersuchung der Sensibilität umfasst die Kontrolle der Oberflächensensibilität mit Erfassung der Berührungs-,

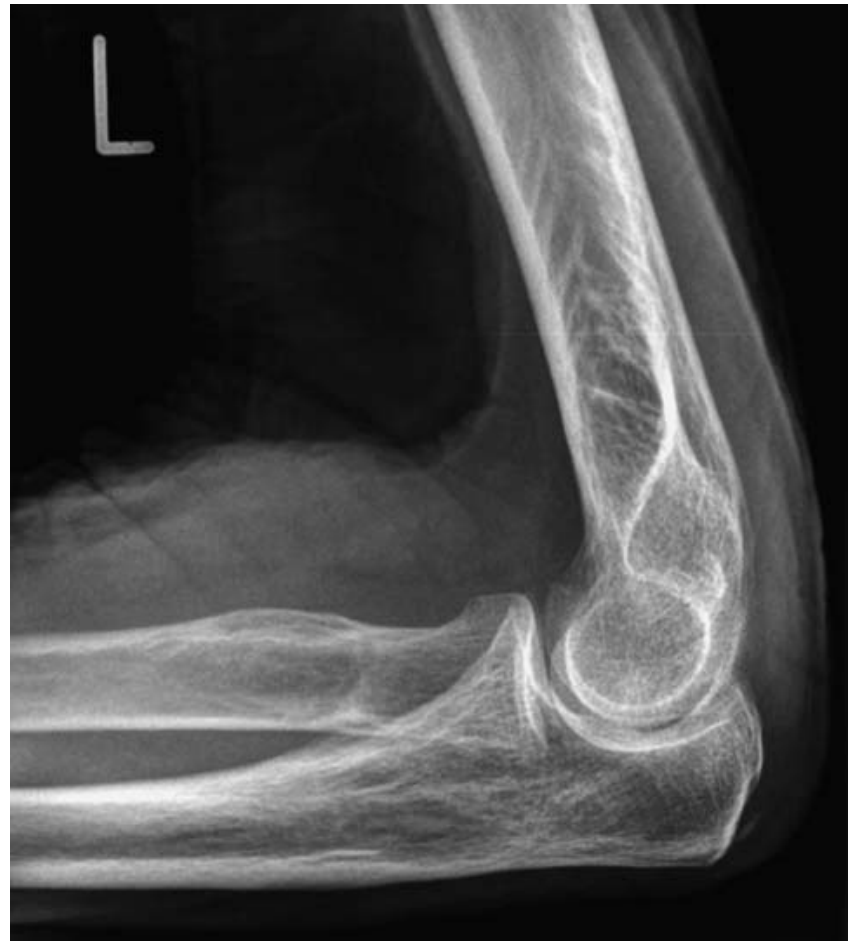

Abb. 5 Seitliche Röntgenaufnahme.
Schmerz- und Temperaturempfindung. Im Ellenbogenbereich erfolgt die nervale Versorgung durch 4 Nerven:

- N. axillaris (C5): lateraler Oberarm

- N. musculocutaneus (C6): medialer Oberarm

- N. cutaneus antebrachii lateralis (C8): lateraler Unterarm

- N. cutaneus antebrachii medialis (Th1): medialer Unterarm

\section{Kinder und Jugendliche}

Bei Ellenbogenverletzungen im Kindesund Jugendalter sollte von einer genauen und möglicherweise schmerzhaften Untersuchung abgesehen werden.

Die Inspektion und Anamnese, ggf. auch der begleitenden Eltern, sollten ausreichend sein, um eine Röntgendiagnostik bei Frakturverdacht zu veranlassen. Die Prüfung der Peripherie ist jedoch unerlässlich. Eine grob neurologische Untersuchung von Motorik und Sensibilität und Durchblutung sollte zum Ausschluss von Gefäß- und Nervenverletzungen durchgeführt werden.

\section{Bildgebung am Ellenbogen}

Eine eingehende Anamnese und klinische Untersuchung führt zu einer Verdachtsdiagnose, die durch eine geeignete Bildgebung bestätigt wird. Dafür ist die Kenntnis der zur Verfügung stehenden
Methoden und deren korrekte Interpretation der nächste wichtige Schritt zur Planung der operativen Versorgung.

\section{Konventionelle Bildgebung}

\section{Standardaufnahmen}

Ellenbogen a.-p.

Der Patient sitzt neben dem Untersuchungstisch. Das Ellenbogengelenk liegt mit gestrecktem, supiniertem Unterarm auf der Röntgenkasette. Der Zentralstrahl liegt im Lot auf der beugeseitigen Ellenbogengelenk- und Kassettenmitte. Der Untersucher erkennt auf der Röntgenaufnahme den orthograd getroffenen Gelenkspalt. Der Kubitalwinkel beträgt normalerweise $5-15^{\circ}$ [5] (Abb. 4).

\section{Ellenbogen lateral}

Der Patient sitzt neben dem Untersuchungstisch. Der Ellenbogen ist rechtwinkelig gebeugt und wird auf Höhe des Schultergelenks gelagert. Der Daumen ist nach oben gerichtet. Bei exakter Einstelltechnik projizieren sich die Humeruskondylen übereinander [5] (Abb. 5). 


\section{Spezialaufnahmen}

Radiuskopfzielaufnahme

In unserer Klinik wird der Arm wie bei der seitlichen Aufnahme gelagert. Der Zentralstrahl verläuft jedoch in einem Winkel von $45^{\circ}$ durch den Radiuskopf. Durch diese Röntgeneinstellung kommt der Radiuskopf überlagerungsfrei zur Darstellung [5] (Abb. 6).

\section{Sulcus-ulnaris-Aufnahme}

Die Rückseite des Unterarms liegt der Röntgenkassette auf. Der Ellenbogen wird so weit wie möglich flektiert. Die Röntgenröhre wird um $10^{\circ}$ gekippt. In dieser Aufnahme kommen beide Epikondylen sowie der Sulcus nervi ulnaris überlagerungsfrei zur Darstellung [5].

\section{Magnetresonanztomografie (MRT)}

Die MRT ist der Goldstandard zur Beurteilung der Ligamente, Sehnen und Muskeln des Ellenbogens. Die Darstellung des Knochens mit der MRT erlaubt es Osteomyelitiden, die Ausdehnung von Knochentumoren und nativ radiologisch okkulte Frakturen zu diagnostizieren.

Für die Bildgebung von Raumforderungen der Weichteile und Nervenengpasssyndrome ist die MRT das diagnostische Mittel der Wahl [6].

Die bildgebende Schnittführung erfolgt in 3 Ebenen: sagittal, koronar und transversal. Standardmäßig liegt der Patient auf dem Rücken mit seitlich gelagertem Arm. Je nach Größe des Patienten kann es erforderlich sein, den Patienten auf dem Bauch zu lagern und den Arm über Kopf zu positionieren. Diese Lagerung wird jedoch in der Regel schlecht toleriert und führt häufig zu Bewegungsartefakten.

\section{Computertomografie (CT)}

Die CT stellt, trotz der breiten Verfügbarkeit, keine Routineuntersuchung des Ellenbogengelenks dar. Die meisten knöchernen Verletzungen können mit den konventionellen Röntgenbildern adäquat beurteilt werden. Bei speziellen Fragestellungen, unklaren Befunden oder komplexen Frakturen ist der Einsatz der CT sinnvoll. Moderne CTs erlauben eine 3-dimensionale Rekonstruktion der Bilddatensätze (Abb. 7).

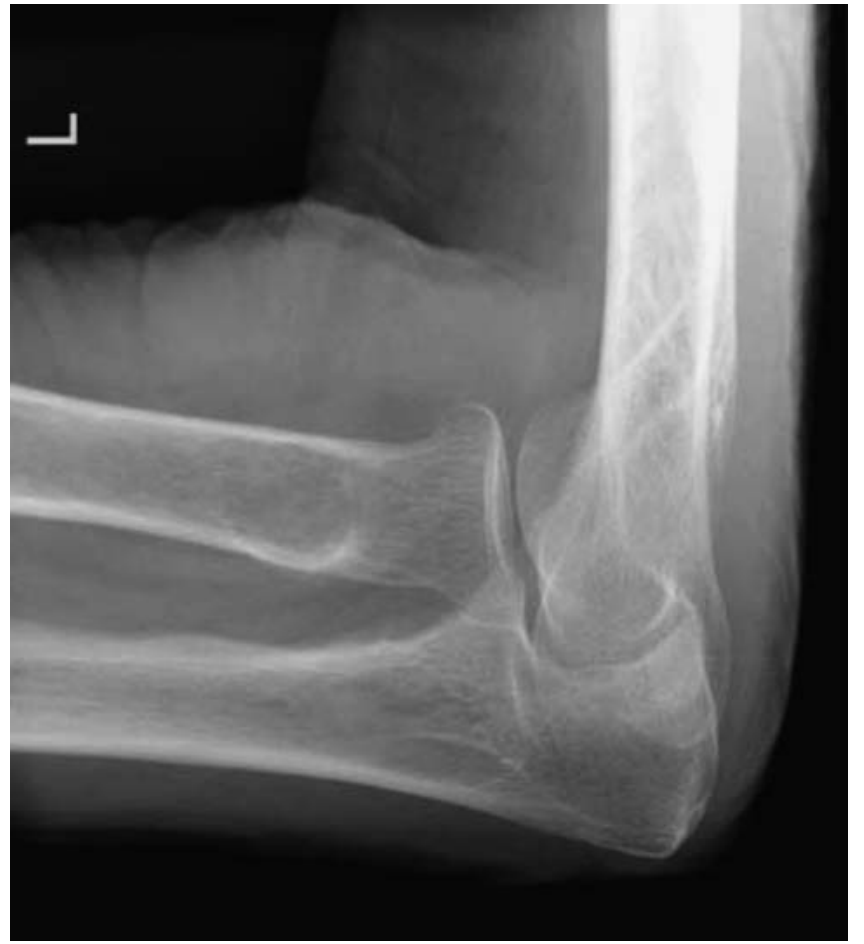

Abb. 6 Radiuskopfzielaufnahme. Der Radiuskopf kommt überlagerungsfrei zur Darstellung.
Ist der Patient in der Lage, die Ellenbogen zu strecken, wird die Untersuchung in Rückenlage durchgeführt. Die Arme werden über den Kopf gestreckt. Ist der Patient nicht in der Lage, den Ellenbogen zu strecken, wird er auf dem Bauch gelagert. Wegen der kurzen Untersuchungszeit ist, im Gegensatz zur MRT, nicht mit Bewegungsartefakten zu rechnen. Oberund Unterarm werden so gelagert, dass sie in einer axialen Ebene zu liegen kommen [5].

\section{Lagerung des Patienten}

Abhängig vom geplanten operativen Eingriff kann das Ellenbogengelenk über 4 Zugangswege erreicht werden: den dorsalen, den lateralen, den medialen und den ventralen Zugang [7]. Die unterschiedlichen Zugänge bedingen unterschiedliche Lagerungen.

Diese sind die Bauchlagerung, die Seitenlagerung und die Rückenlagerung. Bei der Rückenlagerung wird der Arm, je nach Indikation, bedarfsweise auf einen Handtisch ausgelagert. Nicht nur der Zugang, sondern auch der Gesundheitszustand des Patienten müssen bei der Auswahl der Lagerung berücksichtigt werden. Die Lagerung muss bei der Planung des operativen Eingriffs gemeinsam mit der Anästhesie abgesprochen werden. Dem OP-Personal muss mitgeteilt werden, welche Lagerungshilfen benötigt werden. Dadurch lassen sich unnötige Verzögerungen im Operationssaal vermeiden.

\section{Bauchlagerung}

Der Patient wird entweder auf dem Operationstisch selbst gedreht oder, vorteilhaft, aus der Rückenlage von einem 2. Operationstisch über die gesunde Seite auf den eigentlichen Operationstisch gedreht, sodass er dort in Bauchlage zu liegen kommt. Brust und Becken werden mit jeweils einem Kissen unterpolstert. Es ist darauf zu achten, dass das Brustkissen so platziert wird, dass der Kehlkopf des Patienten frei bleibt. Der Kopf wird in einem speziellen Lagerungskissen gelagert. Der verletzte Arm wird im Schultergelenk $90^{\circ}$ flektiert und abduziert. Der Oberarm ruht auf einem Schulterbänkchen. Der Ellenbogen muss so gelagert werden, dass im Ellenbogen eine Flexion von $120^{\circ}$ möglich ist. Am Oberarm wird eine pneumatische Manschette angelegt, die bedarfsweise als Blutsperre benutzt werden kann.

Die Bauchlage ist geeignet, wenn über einen dorsalen Zugang operiert werden soll.

\section{Seitenlagerung}

Der Patient wird so gelagert, dass der zu operierende Arm oben zu liegen kommt. 
Tab. 1 Indikationen für die verschiedenen Zugangswege zum Ellenbogen [10].

\begin{tabular}{|c|c|c|c|}
\hline Dorsaler Zugang & Lateraler Zugang & Medialer Zugang & Ventraler Zugang \\
\hline $\begin{array}{l}\text { Frakturen: } \\
\text { - distaler } \\
\text { Humerus } \\
\text { - Olekranon }\end{array}$ & $\begin{array}{l}\text { Frakturen: } \\
\text { - Epicondylus lateralis } \\
\text { humeri } \\
\text { - Radiuskopf } \\
\text { - Capitulum humeri } \\
\text { - Koronoid }\end{array}$ & $\begin{array}{l}\text { Frakturen: } \\
\text { - Epicondylus } \\
\text { medialis } \\
\text { - Koronoid }\end{array}$ & Arthrolysen \\
\hline Synovektomien & $\begin{array}{l}\text { chronische Radiuskopf- } \\
\text { luxation }\end{array}$ & $\begin{array}{l}\text { mediale Kollateral- } \\
\text { bandruptur }\end{array}$ & $\begin{array}{l}\text { distale Bizepsseh- } \\
\text { nenruptur }\end{array}$ \\
\hline Arthrolysen & Radiuskopfprothese & freier Gelenkkörper & Koronoidfrakturen \\
\hline Arthroplastiken & $\begin{array}{l}\text { Bandplastik bei postero- } \\
\text { lateraler Instabilität }\end{array}$ & $\begin{array}{l}\text { Sulcus-nervi-ulna- } \\
\text { ris-Syndrom }\end{array}$ & $\begin{array}{l}\text { N.-radialis-Kom- } \\
\text { pressionssyndrom }\end{array}$ \\
\hline \multirow{5}{*}{$\begin{array}{l}\text { posttraumatische } \\
\text { Fehlstellungen des } \\
\text { distalen Humerus }\end{array}$} & freier Gelenkkörper & Synovektomien & freie Gelenkkörper \\
\hline & Osteochondrosis dissecans & & \\
\hline & Synovektomien & & \\
\hline & Arthrolysen & & \\
\hline & entzündliche Erkrankungen & & \\
\hline
\end{tabular}
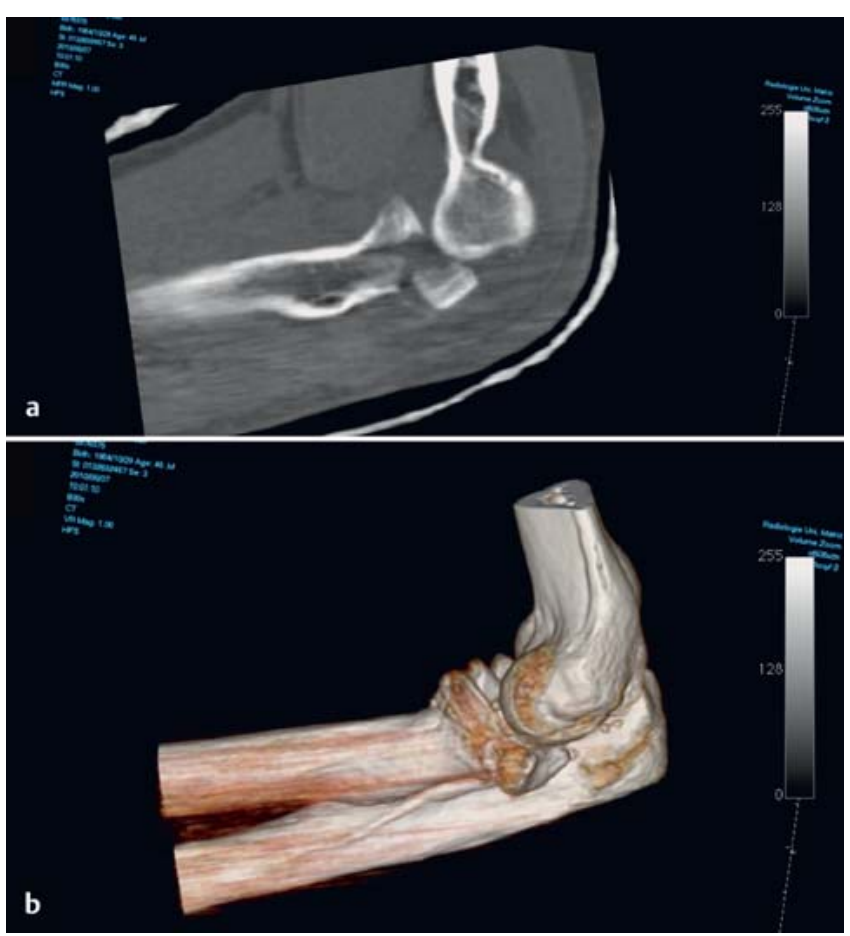

Der Arm wird über eine gepolsterte Rolle gelagert, sodass eine Flexion im Ellenbogengelenk bis $120^{\circ}$ möglich ist. Die Lagerung wird durch gepolsterte Stützen an Brust, Symphyse und Rücken unterstützt. Eine pneumatische Blutsperre wird angelegt und bedarfsweise geschlossen. Die Seitenlage ist geeignet, um das Ellenbogengelenk über den dorsalen Zugang
Abb. $7 \mathbf{a}$ und $\mathbf{b}$

$\mathrm{CT}$ des Ellenbogens mit 3-D-Rekonstruktion bei einer Radiuskopffraktur Typ Mason 3.

Tab. 2 Indikationen zur Ellenbogenarthroskopie $[8,9]$.

\begin{tabular}{l} 
Indikationen zur Ellenbogenarthroskopie \\
\hline freie Gelenkkörper \\
\hline Abtragung von Osteophyten \\
\hline Knorpelschäden \\
\hline Synovitis \\
\hline Adhäsionen \\
\hline Epikondylitis \\
\hline Infektionen \\
\hline chondrale/osteochondrale Frakturen \\
\hline arthroskopisch assistierte Osteosynthesen \\
\hline Instabilität
\end{tabular}

der zu operierende Arm über den Thorax gelagert und in dieser Position von einem Assistenten gehalten. In diesem Fall empfiehlt es sich, die ipsilaterale Schulter mit einem kleinen Lagerungskissen zu unterpolstern oder den Tisch leicht zur Gegenseite zu kippen.

Für den ventralen, lateralen und medialen Zugang wird der Arm frei beweglich auf einem Armtisch gelagert.

Die Indikationen für die jeweiligen $\mathrm{Zu}$ gänge werden in Tab. 1 aufgeführt.

\section{Planung und Vorbereitung einer Ellenbogenarthroskopie}

Die Arthroskopie des Ellenbogengelenks ist zwar wesentlich seltener als die von Knie- oder Schultergelenk, erfreut sich aber zunehmender Beliebtheit $[8,9]$. Die Indikationen für einen arthroskopischen Eingriff am Ellenbogen werden in Tab. 2 genannt. Arthroskopische Eingriffe könne in Bauchlagerung (im eigenen Vorgehen bevorzugt), Seitenlagerung und Rückenlagerung durchgeführt werden.

Nach Lagerung, sterilem Abwaschen und Abdecken werden die knöchernen Landmarken (radialer und ulnarer Epikondylus, Olekranonspitze und Radiuskopf) und die Portale angezeichnet.

zu erreichen. Die Indikationen entsprechen denen der Bauchlagerung.

\section{Rückenlagerung}

Die Rückenlagerung stellt die technisch einfachste Lagerungsmethode dar. In Rückenlage können sämtliche Zugangswege zum Ellenbogen durchgeführt werden. Für einen dorsalen Zugang wird
Die Arthroskopie erfolgt im eigenen Vorgehen in Blutleere. Der 1. Schritt der Arthroskopie stellt das Auffüllen des Gelenks mit elektrolytfreier Lösung (20$50 \mathrm{ml}$ ) dar. 


\section{Planung und Vorbereitung einer Osteosynthese am Ellenbogen}

Nach klinischer und bildgebender Diagnostik einer Fraktur im Bereich des Ellenbogens (Abb. 8) muss der Operateur zunächst entscheiden, ob Spezialuntersuchungen, wie spezielle radiologische Verfahren, erforderlich sind, um die Operation zu planen.

Vor allem bei Mehrfragmentfrakturen im Gelenkbereich bietet sich die CT an, um wichtige Informationen zu erhalten.

Je nach Alter des Patienten kann durch die CT präoperativ entschieden werden, ob eine Rekonstruktion des Gelenks möglich oder eine endoprothetische Versorgung indiziert ist.

Wenn eine Rekonstruktion möglich ist, muss festgelegt werden, welche Osteosynthese (absolute vs. relative Stabilität) notwendig ist, welches Material zum Einsatz kommen soll und welcher $\mathrm{Zu}$ gang die beste Visualisierung der Fraktur bei geringstmöglichem Weichteilschaden erlaubt (Abb. 9) Planungsskizzen können bei der Planung und Vorbereitung vorteilhaft sein [1]. Wichtig ist, schon präoperativ zu überlegen, welches Material man für die Reposition und temporäre Fixierung der Fragmente benötigt. Nur wenn dies klar ist und mit dem OP-Personal kommuniziert wird, können unnötige Verzögerungen intraoperativ vermieden werden. Im eigenen Vorgehen werden diese Aspekte (Lagerung, Narkoseform, Instrumente, Implantate) präoperativ mithilfe der EDV erfasst (Abb. 10) und in einer interdisziplinären Konferenz (Unfallchirurgie/Orthopädie, Anästhesie, OP-Pflege) am Vortag festgelegt.

\section{Literatur}

Porteus M, Südkamp NP, Schatzker J. Preoperative Planning. In: Ruedi TP, Buckley RE, Moran CG, eds. AO Principles of Fracture Management. 2nd ed. Dübendorf, Switzerland: AO Publishing; 2007: 155-163

2 Simmelbauer B, Habermeyer P. [Standardized examination of the elbow]. Unfallchirurg 1996; 99: 548-554

3 Buckup K. Ellenbogengelenk. In: Buckup K, Hrsg. Klinische Tests an Knochen, Gelenken und Muskeln: Untersuchungen - Zeichen Phänomene. 2. Aufl. Stuttgart: Thieme; 2000: 99-108

${ }^{4}$ Jerosch J. Klinische Untersuchung. In: Josten C, Lill H, Hrsg. Ellenbogenverletzungen. 1. Aufl. Darmstadt: Steinkopfverlag; 2002: 13-22

${ }^{5}$ Keitel T, Kahn T. Apparative Diagnostik. In: Josten C, Lill H, Hrsg. Ellenbogenverletzungen. Darmstadt: Steinkopfverlag; 2002: 23-35

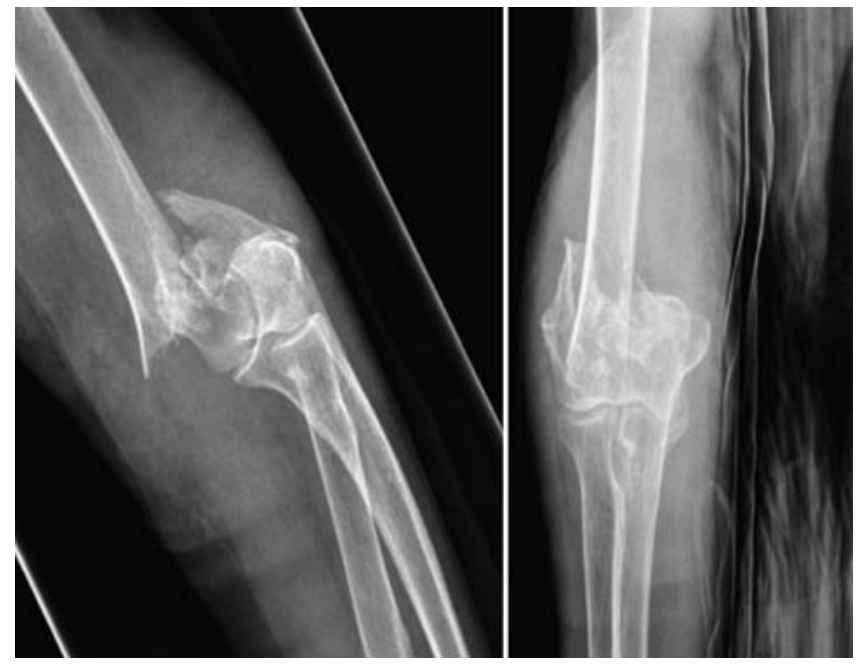

Abb. 8 Dislozierte distale Humerusmehrfragment-Fraktur in a.-p. und seitlicher Projektion.
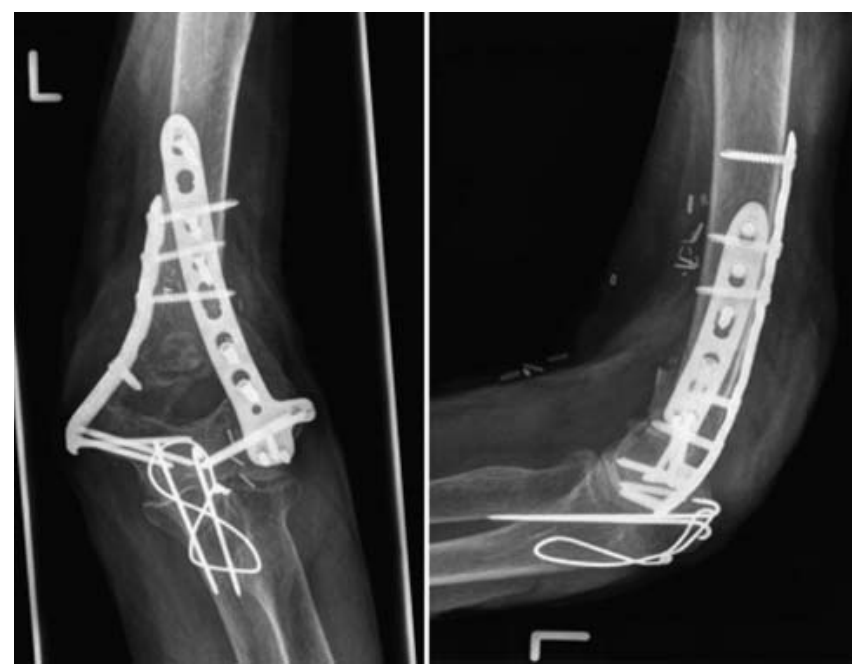

Abb. 9 Postoperative Röntgenbilder in 2 Ebenen: mittels anatomisch vorgeformter winkelstabiler distaler Humerusplatten über eine Olekranonosteotomie versorgte Fraktur. Im ventralen Weichteilgewebe sind Clips der Versorgung der Verletzung der A. brachialis zu sehen.

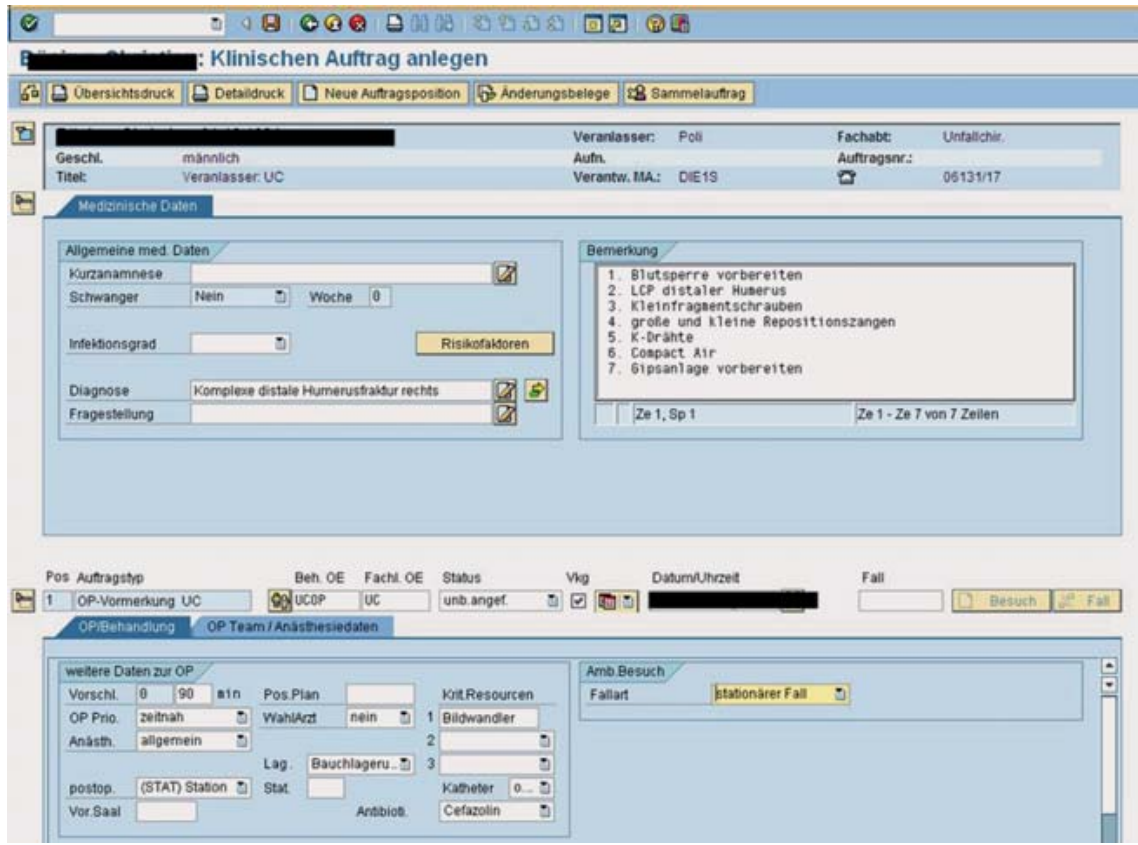

Abb. 10 EDV-gestützte Vorbereitung eines Patienten mit distaler Humerusfraktur. 
${ }^{6}$ Fritz RC. Magnetic Resonance Imaging of the Elbow. In: Baker CL, Plancher KD, eds. Operative Treatment of Elbow Injuries. 1st ed. New York: Springer; 2002: 55-68

7 Cheung EV, Steinmann SP. Surgical approaches to the elbow. J Am Acad Orthop Surg 2009; 17: 325-333

8 Siparsky PN, Kocher MS. Current concepts in pediatric and adolescent arthroscopy. Arthroscopy 2009; 25: 1453-1469
${ }^{9}$ Witt M, Mittlmeier T. [Arthroscopy of the elbow joint]. Unfallchirurg 2007; 110: 953-962

${ }^{10}$ Lill H, Korner J, Glasmacher S. Operative Zugangswege. In: Josten C, Lill H, Hrsg. Ellenbogenverletzungen. 1. Aufl. Darmstadt: Steinkopfverlag; 2002: 51-61

11 Rommens PM. Fractures of the distal Third of the Humerus. In: Flatow EL, Ulrich C, eds. Humerus. 1st ed. Oxford: Butterworth-Heinemann; 1996: 156-183

\section{Dr. med. Tobias Nowak}

Assistenzarzt

Dr. med. Sven-Oliver Dietz

Oberarzt

Prof. Dr. med. Dr. h.c.

Pol M. Rommens

Direktor der Klinik

Klinik und Poliklinik für

Unfallchirurgie

Zentrum für Muskuloskeletale

Chirurgie

Universitätsmedizin Mainz

Johannes Gutenberg Universität

Langenbeckstraße 1

55131 Mainz

sodietz@me.com 